\title{
Castration-Naive Prostate Carcinoma
}

National Cancer Institute

\section{Source}

National Cancer Institute. Castration-Naive Prostate Carcinoma. NCI Thesaurus. Code C148536.

Advanced prostatic carcinoma which was not previously treated with androgendeprivation therapy. 